\title{
On Multivariate Chromatic Polynomials of Hypergraphs and Hyperedge Elimination
}

\author{
Jacob A. White* \\ Mathematical Sciences Research Institute \\ Berkeley, California, USA \\ jawhite@msri.org
}

Submitted: Feb 9, 2011; Accepted: Jul 22, 2011; Published: Aug 5, 2011

Mathematics Subject Classifications: 05C31, 05C15, 05C65

\begin{abstract}
In this paper we introduce multivariate hyperedge elimination polynomials and multivariate chromatic polynomials for hypergraphs. The first set of polynomials is defined in terms of a deletion-contraction-extraction recurrence, previously investigated for graphs by Averbouch, Godlin, and Makowsky. The multivariate chromatic polynomial is an equivalent polynomial defined in terms of colorings, and generalizes the coboundary polynomial of Crapo, and the bivariate chromatic polynomial of Dohmen, Pönitz and Tittman. We prove that specializations of these new polynomials recover polynomials which enumerate hyperedge coverings, matchings, transversals, and section hypergraphs, all weighted according to certain statistics. We also prove that the polynomials can be defined in terms of Möbius inversion on the partition lattice of a hypergraph, and we compute these polynomials for various classes of hypergraphs. We also consider trivariate polynomials, which we call the hyperedge elimination polynomial and the trivariate chromatic polynomial.
\end{abstract}

\section{Introduction}

The chromatic polynomial of a graph enumerates the number of proper $k$-colorings of a graph. It was originally introduced by Birkhoff [BL46], who hoped that understanding this polynomial could lead to a proof of the four-color theorem. The chromatic polynomial was generalized by Tutte to give a two-variable polynomial, now called the Tutte polynomial ([Tut84], [Tut47]). The Tutte polynomial was defined for matroids in general

*The author was partially supported by an NSF grant DMS-0932078, administered by the Mathematical Sciences Research Institute while the author was in residence at MSRI during the Complementary Program, Fall 2010. This work began during the visit of the author to MSRI and we thank the institute for its hospitality. 
by Crapo [Cra69], and has many wonderful enumerative properties. A good survey of these polynomials appears in Matroids and their Applications [BO92].

There has been a recent resurgence in the study of graph polynomials. Prominent examples include the interlace polynomials, matching polynomials, independent set polynomials, and the edge elimination polynomial. A recent survey regarding graph polynomials is given in [EMM11a] and [EMM11b]. This edge elimination polynomial is the main motivation for this paper. It was introduced by Averbouch, Godlin, and Makowsky [AGM10]. It is defined recursively in terms of three graph-theoretic operations - deletion, contraction, and extraction. Extraction does not appear to be a matroidal operation. In fact, not all trees on $n$ vertices have the same edge elimination polynomial, despite having isomorphic cycle matroids.

There are two major purposes of this present paper. The first is to study the hyperedge elimination polynomial, a generalization of the edge elimination polynomial to hypergraphs. Since it makes sense to define deletion, contraction, and extraction for hypergraphs, it also makes sense to prove results in this level of generality. A good resource for learning about hypergraphs is the book by Berge [Ber73].

The second is to introduce the multivariate chromatic polynomial. This polynomial is equivalent to the hyperedge elimination polynomial, as both can be obtained from each other by substitution. Moreover, we prove several results for the multivariate chromatic polynomial, which generalize known results about the coboundary polynomial of Crapo [Cra69], and the bivariate chromatic polynomial of Dohmen, Pönitz, and Tittman [DPT03].

We show that certain evaluations of the hyperedge elimination polynomial or multivariate chromatic polynomial give polynomials that enumerate matchings, stable sets, hyperedge coverings, and section hypergraphs. The first two results are extensions of known results, but the latter two are new. Also, the Tutte polynomial is a specialization of these polynomials. We also give a subset expansion formula for the hyperedge elimination polynomial, generalizing the work of Averbouch et al [AGM10], as well as a Möbius inversion formula, generalizing known results for the coboundary polynomial (appears in [BO92]) and the bivariate chromatic polynomial [DPT03]. Furthermore, we are able to give formulas for the multivariate chromatic polynomial for particular classes of hypergraphs.

\section{Review of Hypergraph Terminology}

A hypergraph is a pair $(V, E, I)$, where $V$ is a finite set of vertices, and $E=\left\{e_{i}: i \in\right.$ $\left.I, \emptyset \neq e_{i} \subseteq V\right\}$ is a collection of hyperedges. We will often abuse notation and refer to $(V, E)$ as a hypergraph, with an understanding that the hyperedges are indexed by some set $I$. Note that we can have $i \neq j \in I$ with $e_{i}=e_{j}$. That is, we are allowing multiple hyperedges in our hypergraphs. In such a case, $e_{i}$ and $e_{j}$ are said to be parallel. A $k$-edge is an hyperedge with $k$ vertices. We are allowing 1-edges, which in the context of coloring is equivalent to the notion of loops in a graph. Let $E_{0}$ denote the hypergraph 
with no vertices or hyperedges. Let $E_{1}$ denote the hypergraph with only one vertex, and no hyperedges.

Given a hypergraph $H$, there are two notions of induced subgraph. Given a subset $A$ of vertices, a subhypergraph is the hypergraph $H_{A}=\left(A,\left\{e_{i} \cap A: e_{i} \cap A \neq \emptyset\right\}\right)$. Note that of course that the new index set for hyperedges is $\left\{i \in I: e_{i} \cap A \neq \emptyset\right\}$. A vertex section hypergraph is the hypergraph $H \times A=\left(A,\left\{e_{i}: e_{i} \subseteq A\right)\right.$. Let $V=\{1,2,3,4\}$, and consider a hypergraph $H$ with edges $e_{1}=\{1,2,3\}$ and $e_{2}=\{2,4\}$. Then the subhypergraph of $H$ induced by the vertex set $A=\{2,3,4\}$ has edges $e_{1} \cap A=\{2,3\}$ and $e_{2}=\{2,4\}$, while the vertex section hypergraph $H \times A$ only has the edge $e_{2}=\{2,4\}$.

Given a subset $J \subseteq I$, let $E_{J}=\left\{e_{j}: j \in J\right\}$, we let $H_{J}=\left(V, E_{J}\right)$ denote the partial hypergraph, and the hyperedge section hypergraph $H \times J$ has hyperedge set $E_{J}$ and vertex set $\cup_{i \in J} e_{i}$. Note that in context one should be able to see the difference between partial hypergraph and subhypergraph. Usually the phrase section hypergraph appears in the literature refering only to vertex section hypergraph. It turns out that the summation definition of the hyperedge elimination polynomial mentioned in this paper can be most easily defined using the notions of partial hypergraph and hyperedge section hypergraph.

Given two hypergraphs, $H=(V, E, I)$ and $H^{\prime}=\left(V^{\prime}, E^{\prime}, I^{\prime}\right)$ such that $V \cap V^{\prime}=\emptyset$, $I \cap I^{\prime}=\emptyset$, we define their disjoint union, $H \sqcup H^{\prime}$, to be the hypergraph $\left(V \cup V^{\prime}, E \cup E^{\prime}, I \cup I^{\prime}\right)$.

Let $H$ be a hypergraph, and let $e_{i}$ be a hyperedge. The deletion is the hypergraph $H-e_{i}=\left(V,\left\{e_{j}: j \neq i\right\}\right)$. The extraction $H \dagger e_{i}$ is the hypergraph $\left(V \backslash e_{i},\left\{e_{j}: e_{j} \cap e_{i}=\emptyset\right\}\right)$. The contraction $H / e$ is obtained from $H \dagger e_{i}$ by adding one new vertex $v_{i}$, and hyperedges $\left\{e_{j}^{\prime}: i \neq j, e_{j} \cap e \neq \emptyset, e_{j}^{\prime}=\left(e_{j} \backslash e_{i}\right) \cup\left\{v_{i}\right\}\right)$. We shall refer to hyperedges $e_{j}^{\prime}$ as the partially contracted hyperedges. We are interested in studying hypergraph polynomials that can be defined recursively in terms of these three operations. The most general polynomial satisfying such a recurrence will be called the hyperedge elimination polynomial. An example of deletion, contraction, and extraction is given in Figure 1. When $H$ is a graph (all hyperedges are 2-edges), deletion and contraction are already familiar operations, and extraction corresponds to deleting an edge and its incident vertices.

A chain is a sequence $v_{0}, e_{1}, v_{1}, \ldots, e_{k}, v_{k}$, where $v_{i} \in e_{i}$ for $1 \leq i \leq k, v_{i} \in e_{i+1}$ for $0 \leq i \leq k-1$, and $e_{1}, \ldots, e_{k}$ are hyperedges. If the hyperedges are all distinct, we obtain a path. If $k>2$ and $v_{0}=v_{k}$, we call the path a cycle. We say that a hypergraph is connected if for every two vertices $u$ and $v$ there exists a path with $v_{0}=u, v_{k}=v$. As with graphs, a hypergraph decomposes into connected components. Let $k(H)$ denote the number of connected components of a hypergraph.

The decision to study multivariate polynomials is motivated by the works of Zaslavsky [Zas92] and Bollobás and Riordan [BR99]. Relationships between graph polynomial sometimes have a multivariate analogue that is easier to prove. For hypergraphs, there are at least two more reasons to focus on studying multivariate polynomials, with indeterminates for each hyperedge. First, given a set of indeterminates $w_{0}, w_{1}, \ldots$, we can make the substitution $t_{e}=w_{|e|}$, and obtain new polynomials. Secondly, we can do a further substitution, replacing $w_{i}$ with $w^{i}$ for some fixed indeterminate $w$. These resulting polynomials cannot be obtained from the hyperedge elimination polynomials for general hypergraphs, yet they contain some very refined data regarding the structure of a graph. 

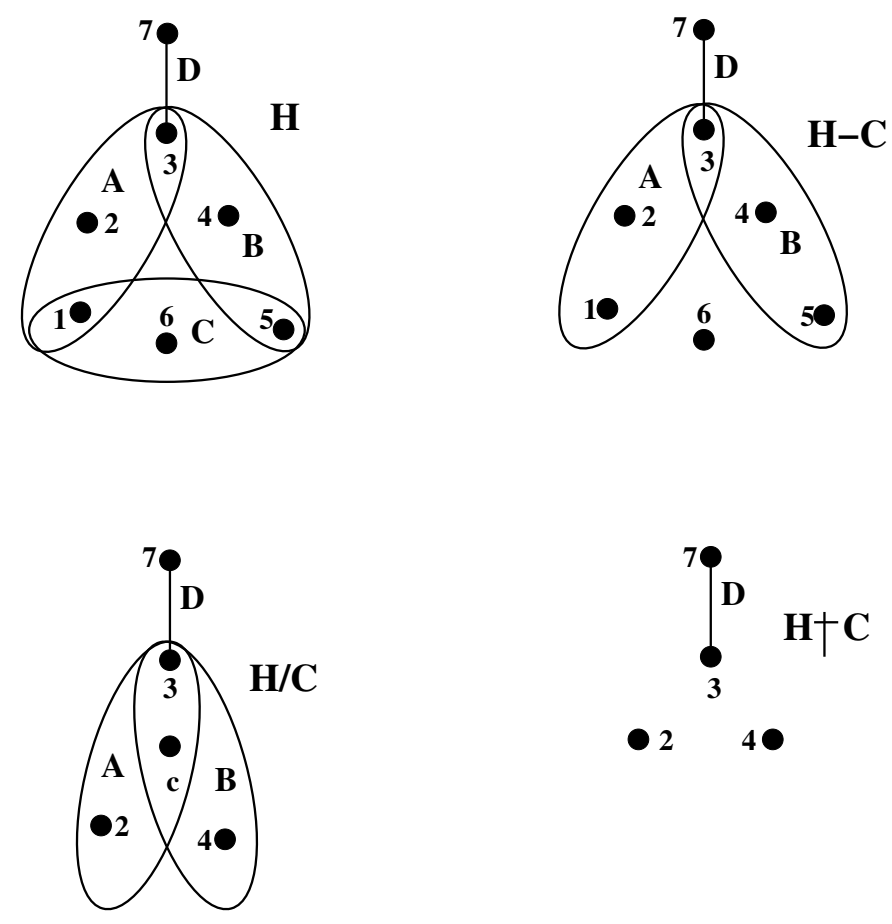

Figure 1: An example of deletion, contraction, and extraction. $H$ has vertices $1,2,3,4,5,6,7$, and hyperedges $A=\{1,2,3\}, B=\{3,4,5\}, C=\{1,5,6\}$, and $D=\{3,7\}$.

Also, there are two notions of hypergraph isomorphism. Two hypergraphs $H=$ $(V, E, I)$ and $H^{\prime}=\left(V^{\prime}, E^{\prime}, I^{\prime}\right)$ are said to be isomorphic if there exists bijections $\varphi: V \rightarrow$ $V^{\prime}$ and $\pi: I \rightarrow I^{\prime}$ such that $v \in e_{i}$ if and only if $\varphi(v) \in e_{\pi(i)}^{\prime}$, for all $v \in V, e_{i} \in E, e_{\pi(i)}^{\prime} \in E^{\prime}$. If $I=I^{\prime}$ and $\pi$ is the identity map, then we refer to $H$ and $H^{\prime}$ are strongly isomorphic. A hypergraph invariant is a function $f$ on graphs such that $f(H)=f\left(H^{\prime}\right)$ whenever $H$ and $H^{\prime}$ are isomorphic. If $f$ is such that $f(H)=f\left(H^{\prime}\right)$ whenever $H$ and $H^{\prime}$ are strongly isomorphic, we refer to $f$ as an edge-labeled hypergraph invariant (as it depends on the index set of $E$ ). All multivariate polynomials considered in this paper are edge-labeled hypergraph invariants. Moreover, the hyperedge elimination polynomial, and its substitutions, are hypergraph invariants.

\section{A List of Interesting Polynomials}

In this section, we define several polynomials involving combinatorial aspects of hypergraphs that are often studied. Most of these polynomials generalize to well-known graph polynomials, but a few of these polynomials are actually new. Throughout, fix a hypergraph $H$ with vertex set $V$ and hyperedge set $E$. Let $n$ be the number of vertices, $m$ be the number of hyperedges. Given any set $F \subseteq E$, let $m_{*}(F)=\sum_{e_{i} \in F}\left|e_{i}\right|$ (note that certain authors use $m_{*}(F)$ to denote the summation $\left.\sum_{e_{i} \in F}\left(\left|e_{i}\right|-1\right)\right)$. 
A matching in $H$ is a set $F \subseteq E$ of hyperedges such that $e_{i} \cap e_{j}=\emptyset$ for all $e_{i} \neq e_{j} \in F$, $i \neq j$. The multivariate matching polynomial is defined by

$$
\mu(H ; x, \mathbf{y})=\sum_{M} x^{n-m_{*}(M)} \prod_{e \in M} y_{e}
$$

where the summation is over all matchings of $H$. When we set $y_{e}=y$ for all $e$, we obtain the bivariate matching polynomial $\mu(H ; x, y)$. This generalizes the bivariate matching polynomial studied by Averbouch et al [AGM10]. For graphs, the substitutions $x=1$ and $y_{u v}=y_{u v} x_{u} x_{v}$ results in the multivariate polynomial originally introduced by Heilman and Lieb [HL72]. So this is one case where we see that it is natural to consider the multivariate version of a polynomial.

A hyperedge covering is a collection of hyperedges $F \subseteq E$ such that $\cup_{e \in F} e=V$. That is, every vertex of $H$ lies on some hyperedge in the covering. A vertex is exposed if it is contained in no hyperedges. The multivariate hyperedge covering polynomial is defined by

$$
\kappa(H ; x, y, \mathbf{t})=\sum_{C} x^{|C|} y^{k\left(\left.H\right|_{C}\right)} \prod_{e \in C} t_{e},
$$

where the sum is over all hyperedge coverings $C$, and the polynomial is 0 if $H$ has an exposed vertex. If we set $t_{e}=1$ for all $e$, we obtain the hyperedge covering polynomial $\kappa(H ; x, y)$. This polynomial does not appear to have been studied in the literature.

A transversal is a set $S \subseteq V$ such that $S \cap e \neq \emptyset$ for all $e \in E$. The transversal polynomial is defined by $\tau(H ; x)=\sum_{S} x^{|S|}$ where the sum is over all transversals of the hypergraph $H$. When $H$ is a graph, this polynomial is sometimes known as the vertex-cover polynomial or independent set polynomial.

The multivariate section polynomial of a hypergraph is defined by

$$
S(H ; x, \mathbf{y})=\sum_{S \subseteq V} x^{n-|S|} \prod_{e \in E(h \times S)} y_{e} .
$$

When $y_{e}=y$ for all $e$, we obtain the section polynomial $S(H ; x, y)$. For graphs, these polynomials enumerate induced subgraphs by number of vertices and edges, and do not appear to have been studied. Note that setting $y_{e}=0$ for all $e$ recovers the transversal polynomial. Also consider the substitution $y_{e}=y_{|e|}$. In the resulting polynomial, the coefficient of $x^{i} y_{1}^{i_{1}} \cdots y_{m}^{i_{m}}$ is the number of section hypergraphs with exactly $n-i$ vertices and $i_{j}$ hyperedges of size $j$ for all $j$. Also note that the resulting polynomial is a hypergraph invariant.

The multivariate dichromatic polynomial is defined by

$$
Z(H ; x, \mathbf{t})=\sum_{J \subseteq I} x^{k\left(\left.H\right|_{J}\right)} \prod_{j \in J} t_{e_{j}}
$$

(following [GJ10]). Note that for graphs one obtains the multivariate Tutte polynomial defined by Sokal [Sok05]. When we set $t_{e}=t$ for all $e$, we get a polynomial that is 
equivalent to the dichromatic polynomial of a graph. Sokal's multivariate Tutte polynomial is a special case of the polynomials investigated by Zaslavsky [Zas92] and Bollobás and Riordan [BR99]. So we choose to rename $Z(H ; x, \mathbf{t})$ the multivariate dichromatic polynomial.

There are two more polynomials we will define in this paper. The first is the hyperedge elimination polynomial $\xi$, defined to satisfy a recurrence involving deletion, contraction, and extraction. The other polynomial is the multivariate chromatic polynomial $P$, which is a generalization of the well-known chromatic polynomial. These two polynomials differ only by substitutions, and hence are equivalent polynomials. Moreover, all the polynomials of this section are all substitutions, up to prefactors, of the hyperedge elimination polynomial (and the multivariate chromatic polynomial). Table 3 shows the polynomials defined in this section, and the corresponding substitutions involved to obtain them from $\xi$ and $P$.

\begin{tabular}{|l|c|c|}
\hline & Polynomial & Substitution \\
\hline Hyperedge Coverings & $\kappa(H ; x, y)$ & $\xi(H ; 0, x, x y)$ \\
\hline & $\kappa(H ; x, y, \mathbf{t})$ & $\xi(H ; 0, x, x y, \mathbf{t})$ \\
\hline Matchings & $\mu(H ; x, y)$ & $\xi(H ; x, 0, y)$ \\
\hline & $\mu(H ; x, \mathbf{y})$ & $\xi(H ; x, 0,1, \mathbf{y})$ \\
\hline Partial Hypergraphs & $Z(H ; x, y)$ & $\xi(H ; x, y, 0)$ \\
\hline & $Z(H ; x, \mathbf{t})$ & $\xi(H ; x, 1,0, \mathbf{t})$ \\
\hline colorings & $P(H ; p, q, t)$ & $\xi(H ; q, t-1,(q-p)(t-1))$ \\
\hline & $P(H ; p, q, \mathbf{t})$ & $\xi(H ; q, 1, p-q, \mathbf{t}-1)$ \\
\hline Section Hypergraphs & $S(H ; x, y)$ & $P(H ; 1, x+1, y)$ \\
\hline & $S(H ; x, \mathbf{y})$ & $P(H ; 1, x+1, \mathbf{y})$ \\
\hline Transversals & $\tau(H ; x)$ & $P(H ; 1, x+1,0)$ \\
\hline Hyperedge Elimination & $\xi(H ; x, y, z)$ & $P\left(H ; x+\frac{z}{y}, x, y+1\right)$ \\
\hline & $\xi(H ; x, y, z, \mathbf{t})$ & $P\left(H ; x+\frac{z}{y}, x, y \mathbf{t}+1\right)$ \\
\hline
\end{tabular}

Table 1: Relationships between some polynomials, $\xi$, and $P$

\section{The Hyperedge Elimination Polynomial}

In this section, we define the hyperedge elimination polynomial. Given a hypergraph $H=(V, E, I)$, for disjoint sets $J, K \subseteq I$, we refer to $(J, K)$ as a vertex disjoint pair if $e \cap f=\emptyset$ for all $e \in E_{J}, f \in E_{K}$.

Definition 1. Let $\xi(H ; x, y, z)$ be defined by

$$
\xi(H ; x, y, z)=\sum_{(I, J)} x^{k\left(H_{I \sqcup J}\right)-k(H \times J)} y^{|I|+|J|-k(H \times J)} z^{k(H \times J)}
$$

where the sum is over vertex disjoint pairs $(I, J)$. 
Proposition 1. $\xi(H ; x, y, z)$ satisfies the following:

1. $\xi\left(E_{0} ; x, y, z\right)=1$,

2. $\xi\left(E_{1} ; x, y, z\right)=x$,

3. $\xi\left(H \sqcup H_{2} ; x, y, z\right)=\xi\left(H_{1} ; x, y, z\right) \cdot \xi\left(H_{2} ; x, y, z\right)$ whenever $H=H_{1} \sqcup H_{2}$,

4. for any $e \in E(H)$, we have

$\xi(H ; x, y, z)=\xi(H-e ; x, y, z)+y \xi(H / e ; x, y, z)+z \xi(H \dagger e ; x, y, z)$.

We actually prove a similar recurrence of the multivariate hyperedge elimination polynomial in a later section. The above theorem follows through specialization.

Theorem 2. Let $f$ be a function from hypergraphs to some integral domain $R$, such that $f$ is invariant under hypergraph isomorphism, and $f$ satisfies a recurrence with parameters $\alpha, \beta, \gamma, \delta \in R$ subject to:

1. $f\left(E_{0}\right)=1$,

2. $f\left(E_{1}\right)=\alpha$,

3. $f\left(H_{1} \sqcup H_{2}\right)=f\left(H_{1}\right) \cdot f\left(H_{2}\right)$ whenever $H=H_{1} \sqcup H_{2}$,

4. for any $e \in E(H)$, we have $f(H)=\beta f(H-e)+\gamma f(H / e)+\delta f(H \dagger e)$.

Then either:

1. $\delta=0$ and $f(H)=\beta^{m(H)} \xi\left(H ; \alpha, \frac{\gamma}{\beta}, 0\right)$,

2. $\beta=1$ and $f(H)=\xi(H ; \alpha, \gamma, \delta)$,

3. $f(H)=\alpha^{n(H)}=\xi(H ; 1, \alpha, 0)$.

Proof. If $\delta=0$ or $\beta=1$ we see that the corresponding evaluation of $\xi$ yields the same recursion as $f$, and hence the equality holds.

So assume $\delta \neq 0$ and $\beta \neq 1$. Let $H$ be a hypergraph, let $v$ be a vertex of $H$. Consider two new vertices $y, z$ that are not vertices of $H$, and construct a new hypergraph $G$, by adding vertices $y, z$ to $H$, and hyperedges $e$ with vertex set $v y$ and $f$ with vertex set $y z$.

First, eliminate hyperedge $e$, then eliminate hyperedge $f$ :

$$
\begin{aligned}
f(G)= & \beta f(G-e)+\gamma f(G / e)+\delta f(G \dagger e) \\
= & \beta(\beta f(G-e-f)+\gamma f(G-e / f)+\delta f(G-e \dagger f)) \\
& +\gamma(\beta f(G / e-f)+\gamma f(G / e / f)+\delta f(G / e \dagger f))+\delta f(G \dagger e) \\
= & \left(\beta^{2} \alpha^{2}+2 \alpha \beta \gamma+\beta \delta+\gamma^{2}\right) f(H)+(\alpha \delta+\gamma \delta) f(H-v)
\end{aligned}
$$

Instead, first eliminate hyperedge $f$, then hyperedge $e$ to obtain:

$$
f(G)=\left(\beta^{2} \alpha^{2}+2 \alpha \beta \gamma+\delta+\gamma^{2}\right) f(H)+(\alpha \beta \delta+\gamma \delta) f(H-v)
$$


Thus we obtain:

$$
\alpha \delta f(H-v)+\beta \delta f(H)=\delta f(H)+\alpha \beta \delta f(H-v)
$$

or equivalently:

$$
(1-\beta) \delta \alpha f(H-v)=(1-\beta) \delta f(H)
$$

Since $\delta \neq 0, \beta \neq 1$, and $R$ is an integral domain, we have $\alpha f(H-v)=f(H)$ for all hypergraphs $H$ and vertices $v$. Hence an inductive argument on $n$ yields that $f(H)=$ $\alpha^{n(H)}$.

Thus, any sort of function that obeys the hyperedge elimination recursion and is a hypergraph invariant must be, up to prefactor, an evaluation of the hyperedge elimination polynomial. Note that this argument and result for graphs was previously given by Averbouch et al. [AGM10].

\section{The Multivariate Hyperedge Elimination Polyno- mial}

Now we define the multivariate hyperedge elimination polynomial. This is a hypergraph extension of the labeled hyperedge elimination polynomial, and we denote it $\xi(G ; x, y, z, \mathbf{t})$.

Definition 2. Let $\xi(H ; x, y, z, \boldsymbol{t})$ be defined by

$$
\xi(H ; x, y, z, \boldsymbol{t})=\sum_{(I, J)} x^{k\left(H_{I \sqcup J}\right)-k(H \times J)} y^{|I|+|J|-k(H \times J)} z^{k(H \times J)} \prod_{i \in I \sqcup J} t_{e_{i}}
$$

where the sum is over vertex disjoint pairs $(I, J)$.

Theorem 3. $\xi(H ; x, y, z)$ satisfies the following:

1. $\xi\left(E_{0} ; x, y, z, \boldsymbol{t}\right)=1$,

2. $\xi\left(E_{1} ; x, y, z, \boldsymbol{t}\right)=x$,

3. $\xi\left(H_{1} \sqcup H_{2} ; x, y, z, \boldsymbol{t}\right)=\xi\left(H_{1} ; x, y, z, \boldsymbol{t}_{1}\right) \cdot \xi\left(H_{2} ; x, y, z, \boldsymbol{t}_{2}\right)$ whenever $H=H_{1} \sqcup H_{2}$, where $\boldsymbol{t}_{j}=\left\{t_{e_{i}}: e_{i} \in E\left(H_{j}\right)\right\}$,

4. for any $e \in E(H)$, we have

$$
\begin{aligned}
\xi(H ; x, y, z, \boldsymbol{t}) & =\xi\left(H-e ; x, y, z, \boldsymbol{t}_{\neq e}\right) \\
& +y t_{e} \xi\left(H / e ; x, y, z, \boldsymbol{t}_{\neq e}\right) \\
& +z t_{e} \xi\left(H \dagger e ; x, y, z, \boldsymbol{t}_{\perp e}\right)
\end{aligned}
$$

where $\boldsymbol{t}_{\neq e}=\left\{t_{f}: f \in E(H-e)\right\}$ and $\boldsymbol{t}_{\perp e}=\left\{t_{f}: f \in E(H \dagger e)\right\}$. 
Proof. Let $(I, J)$ be a vertex disjoint pair. Let $p(H ; I, J)=$ $x^{k\left(H_{I \sqcup J}\right)-k(H \times J)} y^{|I|+|J|-k(H \times J)} z^{k(H \times J)} \prod_{i \in I \sqcup J} t_{e_{i}}$. Observe that $e \notin E_{I \sqcup J}$ if and only if $(I, J)$ is a vertex disjoint pair for $H-e$. In such a case we see that $p(H-e ; I, J)=p(H ; I, J)$. Now suppose $e \in E_{J}$, say $e_{j}$, and that it is in its own component. Then $(I, J-j)$ is a vertex disjoint pair for $H \dagger e$, and moreover $p(H ; I, J)=z t_{e} p(H \dagger e ; I, J-j)$. Suppose $e_{j}$ is not in an isolated component. Then $(I, J-j)$ is a vertex disjoint pairt for $H / e$ and $p(H ; I, J)=y t_{e} p(H / e ; I, J-j)$. Moreover, this covers all vertex disjoint pairs $(I, J)$ of $H / e$ for which $(I, J+j)$ is vertex disjoint for $H$ but $(I+j, J)$ is not. Now suppose $e=e_{i}, i \in I$. Then $(I-i, J)$ is vertex disjoint for $H / e$, and $p(H ; I, J)=p(H / e ; I-i, J)$. This covers all vertex disjoint pairs $(I, J)$ of $H / e$ for which $(I+i, J)$ is a vertex disjoint pair for $H$. Thus, summing over all $(I, J)$, we obtain the result.

Similar to [AGM10], we could study the most general hyperedge elimination recurrence. However, in the end, like Averbouch et al, we are unable to prove that any invariant satsifying the labeled hyperedge elimination recurrence is up to prefactor an evaluation of $\xi(H ; x, y, z, \mathbf{t})$.

\subsection{Substitutions of the Multivariate Hyperedge Elimination Polynomial}

In this section, we consider what happens when we evaluate some of the variables in $\xi(H ; x, y, z, \mathbf{t})$ at 0 . Most of these evaluations extend known results for graphs. Note that $\xi(H ; x, y, z, \mathbf{t})=x^{n}$ when $t_{e}=0$ for all hyperedges $e$.

First, if we evaluate at $z=0$, we see that our hyperedge elimination recurrence only involves deletion and contraction, and thus we end up with the multivariate dichromatic polynomial.

Now we consider setting $y=0$. In this case, our recurrence only involves deletion and extraction. One can check that the resulting recurrence is satisfied by the multivariate matching polynomial $\mu(H ; x, y, \mathbf{t})$. Consider an hyperedge $e$. Any matching not involving $e$ is enumerated in $H-e$. Any matching involving $e$ is enumerated by $y_{e} \mu\left(H \dagger e ; x, \mathbf{y}_{\neq e}\right)$. So $\mu(H ; x, \mathbf{y})=\mu(H ; x, \mathbf{y})+y_{e} \mu\left(H ; x, \mathbf{y}_{\neq e}\right)$. Hence $\xi(H ; x, 0, z, \mathbf{t})=\mu(H ; x, z \mathbf{t})$.

Finally, consider the case $x=0$. This case was not considered by Averbouch et al [AGM10], and actually yields an interesting polynomial for hypergraphs. If $H$ has an isolated vertex, the result is 0 . So suppose $H$ has no isolated vertices. In terms of Equation 1, the only terms that do not vanish correspond to disjoint vertex pairs $(I, J)$ for which $k\left(H_{I \sqcup J}\right)=k(H \times J)$. In such a situation $I=\emptyset$, and $H$ must not have any isolated vertices. Then we see that $k\left(H_{J}\right)=k(H \times J)$, which is true if and only if $E_{J}$ is a hyperedge cover of $H$. Therefore the subset expansion reduces to a summation over hyperedge coverings, and we see that we obtain the hyperedge cover polynomial. Thus $\kappa(H ; x, y, \mathbf{t})=\xi(H ; 0, x, x y, \mathbf{t})$. One could also verify the hyperedge elimination recurrence, and the initial condition $\kappa\left(E_{1}, x, y, \mathbf{t}\right)=0$.

Thus, we have shown the following proposition, which corresponds to the first three rows of Table 3. 
Proposition 4. We have the following identities:

- $Z(H ; x, \boldsymbol{t})=\xi(H ; x, 1,0, \boldsymbol{t})$,

- $\mu(H ; x, \boldsymbol{y})=\xi(H ; x, 0,1, \boldsymbol{y})$,

- $\kappa(H ; x, y, \boldsymbol{t})=\xi(H ; 0, x, x y, \boldsymbol{t})$,

- $Z(H ; x, y)=\xi(H ; x, y, 0)$,

- $\mu(H ; x, y)=\xi(H ; x, 0, y)$,

- $\kappa(H ; x, y)=\xi(H ; 0, x, x y)$.

\subsection{Hyperedge Elimination Polynomial for Paths and Cycles}

Let $P_{m, r}$ be the $r$-uniform elementary path hypergraph with $m$ hyperedges, where $r \geq 2$, $m \geq 1$. That is, $P_{m, r}$ has hyperedges $e_{1}, \ldots, e_{m}$, where each hyperedge has exactly $r$ vertices. Moreover, $\left|e_{i-1} \cap e_{i}\right|=1$ for $2 \leq i \leq m$, and $\left|e_{i} \cap e_{i+1}\right|=1$ for $1 \leq i \leq m-1$, and these hyperedges sets are otherwise disjoint.

Let $P_{m, r}(x, y, z)=\xi\left(P_{m, r} ; x, y, z\right)$. For fixed $r$, we have thus defined a sequence of polynomials.

Consider applying hyperedge elimination to the hyperedge $e_{1}$. We see that $P_{m, r}-e_{1}$ has $r-1$ isolated vertices, and then $P_{m-1, r}$ as the remaining component. Thus $\xi\left(P_{m, r}-e\right)=$ $x^{r-1} \xi\left(P_{m-1}, r\right)$. Similarly, $\xi\left(P_{m, r} / e\right)=\xi\left(P_{m-1, r}\right)$, and $\xi\left(P_{m, r} \dagger e\right)=x^{r-2} \xi\left(P_{m-2, r}\right)$.

Thus $P_{m, r}(x, y, z)=\left(x^{r-1}+y\right) P_{m-1, r}(x, y, z)+z x^{r-2} P_{m-2, r}(x, y, z)$, for $m>2$, with initial conditions $P_{0, r}(x, y, z)=1$ and $P_{1, r}(x, y, z)=x^{r}+y x+z$. It then follows that the generating function $P_{r}(x, y, z, q)=\sum_{m \geq 0} P_{m, r}(x, y, z) q^{m}$ is given by $\frac{1+x^{r}+y x+z+q\left(x^{r-1}+y\right)}{1-q\left(x^{r-1}+y\right)-q^{2} z x^{r-2}}$.

Let $C_{m, r}$ be the $r$-uniform elementary hypercycle with $m \geq 3$ hyperedges. That is, $C_{m, r}$ is obtained from $P_{m, r}$ by identifying some vertex in $e_{1} \backslash e_{2}$ and $e_{m} \backslash e_{m-1}$. Let $C_{m, r}(x, y, z)=\xi\left(C_{m, r} ; x, y, z\right)$. Then $C_{m, r}(x, y, z)=x^{r-2} P_{m-1, r}(x, y, z)+y C_{m-1, r}(x, y, z)+$ $z x^{2 r-4} P_{m-3, r}(x, y, z)$. This is shown by applying hyperedge elimination to any hyperedge of $C_{m, r}$. One can use this to give a recurrence for $C_{m, r}$ of order 3 . However, we do not take this approach, as it is tedious.

\section{The Multivariate Chromatic Polynomial}

Now we define the multivariate chromatic polynomial, which generalizes the coboundary polynomial and bivariate chromatic polynomial of a graph. Let $q$ be a positive integer. Then a function $f: V(H) \rightarrow[q]$ is refered to as a $q$-coloring. A hyperedge $e_{i}$ is monochromatic if $f(u)=f(v)$ for all $u, v \in e$. The coloring $f$ is proper if it has no monochromatic hyperedge. Let $p \leq q$ be a positive integer. We now view $1, \ldots, p$ as primary colors. A hyperedge $e$ is called primary if $f(u)=f(v) \leq p$ for all $u, v \in e$. In other words, the hyperedge is primary if all the vertices are colored with the same primary color. Given a coloring $f$, let $P(f)$ denote the set of primary edges. 
Let

$$
P(H ; p, q, \mathbf{t})=\sum_{f: V \rightarrow[q]} \prod_{e \in P(f)} t_{e} .
$$

We see that for every pair of positive integers $p \leq q$, this defines a multivariate polynomial in the $m(H)$ variables $\left\{t_{e_{i}}: i \in I\right\}$. However, we will show that $P(H ; p, q, \mathbf{t})$ defines a multivariate polynomial in indeterminates $p, q$ and $\left\{t_{e_{i}}: i \in I\right\}$. We call this polynomial the multivariate chromatic polynomial. Also, we call $P(H ; p, \mathbf{t})=P(H ; p, p, \mathbf{t})$ the multivariate coboundary polynomial. That is, when $H$ is a graph, and we set all $t_{e}=t$, then we obtain the coboundary polynomial introduced by Crapo [Cra69]. We see that $P(H ; p, q)=P(H ; p, q, 0)$ is the bivariate chromatic polynomial defined by Dohmen Pönitz and Tittman [DPT03]. Finally, let $P(H ; p, q, t)$, the trivariate chromatic polynomial be obtained by setting all $t_{e}=t$. Next we prove a theorem that expresses the multivariate chromatic polynomial of $H$ in terms of coboundary polynomials of section hypergraphs of $H$. This generalizes a result of Dohmen et al [DPT03].

Proposition 5. $P(H ; p, q, \boldsymbol{t})=\sum_{S \subseteq V} P(H \times S ; p, \boldsymbol{t})(q-p)^{|V|-|S|}$.

Proof. Let $S$ be a set of vertices, $p \leq q$ be positive integers, and consider the set $P_{S}$ of all $q$-colorings $f$ of $H$ such that $f^{-1}([p])=S$. Let $P(S)=\sum_{f \in P_{S}} \prod_{e \in P(f)} t_{e}$. Given such a coloring $f,\left.f\right|_{S}$ is a $p$-coloring of $H \times S$, and the monochromatic hyperedges of this coloring are the primary hyperedges of $H$. Moreover, there are $(q-p)^{|V|-|S|}$ colorings $f^{\prime}$ such that $f^{\prime-1}([p])=S$ and $\left.f^{\prime}\right|_{S}=\left.f\right|_{S}$. Thus we see that $P(S)=P(H \times S ; p, \mathbf{t})(q-p)^{|V|-|S|}$. Clearly $P(H ; p, q, \mathbf{t})=\sum_{S \subseteq V} P(S)$, and the result follows.

\subsection{Multivariate Section Polynomial of a Hypergraph}

Recall that we defined the multivariate section polynomial by

$$
S(q, \mathbf{t})=\sum_{S \subseteq V} q^{|V|-|S|} \prod_{e \in E(H \times S)} t_{e}
$$

Then the next result may be viewed as a multivariate generalization of the result of Dohmen et al [DPT03].

Theorem 6. $S(H ; q, \boldsymbol{t})=P(H ; 1, q+1, \boldsymbol{t})$.

Proof. We know that $P(H ; 1, q+1, \mathbf{t})=\sum_{S \subseteq V} q^{|V|-|S|} P(H \times S ; 1, \mathbf{t})$, and clearly $P(H ; 1, \mathbf{t})=\prod_{e \in E(H)} t_{e}$ for any hypergraph $\bar{H}$.

Corollary 7. Upon setting $t_{e}=0$ for all e, we obtain: $\tau(H ; x)=P(H ; 1, q+1,0)$.

Note that this corollary was obtained for graphs by Dohmen et al [DPT03]. 


\subsection{A Möbius Inversion Formula for $P$}

Given a partition $\pi$ of $V$, we say that $\pi$ is a connected partition if $H \times S$ is a connected hypergraph for each block $S$ of $\pi$ that is not a singleton. Let $\Pi_{H}$ denote the collection of connected partitions of $H$, ordered by refinement. Observe that $\Pi_{H}$ is an example of a lattice. It has a unique minimum element, corresponding to the partition of $V$ into singletons. It also has a maximum element, corresponding to partitioning $V$ into the vertex sets of the components of $H$.

Given a connected partition $\pi$, and positive integers $p \leq q$, let $f(\pi)$ denote the number of colorings such that:

- If $f(u)>p$ then $u$ is a singleton in $\pi$.

- If $e$ is a primary hyperedge, then $e \subseteq S$ for some block $S$ of $\pi$.

For graphs this definition is equivalent to the definition of $f(\pi)$ given in the paper of Dohmen et al [DPT03]. By abuse of notation, we write $e \subseteq \pi$ to mean the vertex set of $e$ is a subset of some block of $\pi$. Finally, let $k_{1}(\pi)$ denote the number of singleton blocks of $\pi$.

Theorem 8. $P(H ; p, q, \boldsymbol{t})=\sum_{\pi \leq \sigma \in \Pi_{H}} q^{k_{1}(\sigma)} p^{|\sigma|-k_{1}(\sigma)} \mu(\pi, \sigma) \prod_{e: e \subseteq \pi} t_{e}$.

Proof. First, note that $P(H ; p, q, \mathbf{t})=\sum_{\pi \in \Pi_{H}} f(\pi) \prod_{e: e \subseteq \pi} t_{e}$. That is, given a coloring $f$, if we separate color classes into their connected components, and separate all vertices $u$ such that $f(u)>p$ into singletons, we obtain a partition $\pi \in \Pi_{H}$, and moreover, $f$ is counted by $f(\pi)$. Also, the primary edges in $P(f)$ must be the edges contained in blocks of $\pi$. The result follows by noting that the resulting map sending $f$ to $\pi$ is surjective, and for any partition $\pi$, the size of the preimage of this map is $f(\pi)$.

Also, note that

$$
q^{k_{1}(\pi)} p^{|\pi|-k_{1}(\pi)}=\sum_{\sigma \geq \pi} f(\sigma)
$$

By Möbius inversion, we have $f(\pi)=\sum_{\sigma \geq \pi} \mu(\pi, \sigma) q^{k_{1}(\sigma)} p^{|\sigma|-k_{1}(\sigma)}$. Combined with the expression of $P(H ;, p, q, \mathbf{t})$ in terms of $f(\pi)$, the result follows.

This gives a proof that $P(H ; p, q, \mathbf{t})$ is a polynomial. This theorem generalizes known results in the case $t_{e}=0$ [DPT03], and the case $p=q$ [BO92].

\subsection{Relationship between $P$ and $\xi$}

Theorem 9. $P(H ; p, q, \boldsymbol{t})$ satisfies the following:

1. $P\left(E_{0} ; p, q, \boldsymbol{t}\right)=1$,

2. $P\left(E_{1} ; p, q, \boldsymbol{t}\right)=q$,

3. $P\left(H_{1} \sqcup H_{2} ; p, q, \boldsymbol{t}\right)=P\left(H_{1} ; p, q, \boldsymbol{t}\right) \cdot P\left(H_{2} ; p, q, \boldsymbol{t}\right)$ whenever $H=H_{1} \sqcup H_{2}$, 
4. for any $e \in E(H)$, we have

$$
\begin{aligned}
P(H ; p, q, \boldsymbol{t}) & =P\left(H-e ; p, q, \boldsymbol{t}_{\neq e}\right) \\
& +\left(t_{e}-1\right) P\left(H / e ; p, q, \boldsymbol{t}_{\neq e}\right) \\
& +\left(1-t_{e}\right)(q-p) P\left(H \dagger e ; p, q, \boldsymbol{t}_{\perp e}\right)
\end{aligned}
$$

where $\boldsymbol{t}_{\neq e}=\left\{t_{f}: f \in E-e\right\}, \boldsymbol{t}_{\perp e}=\left\{t_{f}: f \in E \dagger e\right\}$.

Proof. The only difficult statement is the hyperedge elimination recurrence. Given a coloring $f$, let $p(H, f)=\prod_{e \in P(f)} t_{e}$. We consider three different types of colorings: if the hyperedge $e$ is primary under the coloring $f$, then we say $f$ is of type 3 . If $e$ is monochromatic but not primary, we say it is of type 2 . Otherwise, we say $f$ is of type 1 . Let $P_{i}(H ; x, y, z, \mathbf{t})$ be the summation previously defined for $P(H ; p, q, \mathbf{t})$, but restricted only to colorings of type $i$. Then $P(H ; p, q, \mathbf{t})=P_{1}(H ; p, q, \mathbf{t})+P_{2}(H ; p, q, \mathbf{t})+P_{3}(H ; p, q, \mathbf{t})$. We also divide colorings of $H / e$ into two types. Such a coloring is of type $a$ if $f\left(v_{e}\right) \leq p$, and is of type $b$ otherwise.

Suppose $f$ is of type 1 . Then $f$ is a coloring of $H-e$ and $p(H-e, f)=p(H, f)$. If $f$ is of type 3 , then we see that $f$ is a coloring of $H-e$ and $f$ induces a coloring on $H / e$ by making $v_{e}$ the same color as the vertices of $e$. Then $p(H, f)=p(H-e, f)+\left(t_{e}-1\right) p\left(H / e, f^{\prime}\right)$. Note that $f^{\prime}$ is a coloring of type $a$. Finally, suppose $f$ is of type 2 , and let $f^{\prime}$ be the induced coloring on $H / e, f^{\prime \prime}$ be the coloring restricted to $V-e$. Then $p(H, f)=$ $p(H-e, f)+\left(t_{e}-1\right) p(H / e, f)+\left(1-t_{e}\right) P\left(H \dagger e, f^{\prime \prime}\right)$. Note that $f^{\prime}$ is a coloring of type $b$.

We see that if we substitute these relations in the summation $P_{1}+P_{2}+P_{3}$, we obtain the result.

Note that this allows us to conclude that $P(H ; p, q, \mathbf{t})$ is a substitution of $\xi(H ; x, y, z, \mathbf{t})$. It is not hard to show that the converse is also true, so these polynomials are equivalent.

Proposition 10. We have the following:

1. $P(H ; p, q, \boldsymbol{t})=\xi(H ; q, 1, p-q, \boldsymbol{t}-1)$,

2. $\xi(H ; x, y, z, \boldsymbol{t})=P\left(H ; x+\frac{z}{y}, x, y \boldsymbol{t}+1\right)$.

\subsection{Multivariate Chromatic Polynomial for Special Classes of Hypergraphs}

Here we study the multivariate chromatic polynomials of complete $r$-uniform hypergraphs, complete $r$-uniform hyperstars, and sunflower hypergraphs. In some sense, this demonstrates some of the beauty of studying multivariate chromatic polynomials: trying to obtain the equivalent expressions from the hyperedge elimination recurrence, or from subset expansion seem unlikely, but the coloring interpretation makes it much simpler.

The complete $r$-uniform hypergraph $K_{n}^{r}$ has vertex set $[n]$ and edges $\left\{i_{1}, \ldots, i_{r}\right\}$ for $1 \leq i_{1}<i_{2}<\cdots<i_{r} \leq n$, one edge for each such $r$-subset of [n]. A set composition $\pi$ 
on $[n]$ is a sequence $S_{0}, \ldots, S_{k}$ of disjoint sets whose union is $[n]$. We let $\ell(\pi)=k+1$ in this case, and write $\pi \models[n]$. Given a set composition, $S_{0}, S_{1}, \ldots, S_{\ell(\pi)-1}$ will be used to denote the sets in the sequence. The following formula holds for any hypergraph:

\section{Proposition 11.}

$$
P(H ; p, q, \boldsymbol{t})=\sum_{\pi \models V(H)}(q-p)^{\left|S_{0}\right|}\left(\begin{array}{c}
p \\
\ell(\pi)-1
\end{array}\right) \prod_{i=1}^{\ell(\pi)-1} \prod_{e_{j} \subset S_{i}} t_{e_{j}} .
$$

Proof. The set $S_{0}$ and the term $(q-p)^{\left|S_{0}\right|}$ corresponds to picking a subset of vertices, and coloring them with the non-primary colors. The rest of the set composition and the term $\left(\begin{array}{c}p \\ \ell(\pi)-1\end{array}\right)$ comes from choosing color classes, and assigning them colors. The final products just come from seeing what edges are monochromatic under the resulting colorings.

Of course, the importance is that for $r$-uniform complete hypergraphs, one can obtain a nice formula for the trivariate chromatic polynomial:

\section{Corollary 12.}

$$
P\left(K_{n}^{r} ; p, q, t\right)=\sum_{\pi \mid=V(H)}(q-p)^{\left|S_{0}\right|}\left(\begin{array}{c}
p \\
\ell(\pi)-1
\end{array}\right) \prod_{i=1}^{\ell(\pi)-1} t^{\left(\begin{array}{c}
\left|S_{i}\right| \\
r
\end{array}\right)}
$$

We say that an $r$-uniform hypergraph is a hyperstar if $\cap_{e \in H} e \neq \emptyset$. Given $v \in V$, $H$ is a complete $r$-uniform hyperstar centered at $v$ if the hyperedge set consists of all $r$-subsets of $V$ containing $v$ (with no parallel hyperedges). Such hypergraphs are unique up to isomorphism, so we define the complete $r$-uniform hyperstar on $[n]$ to consist of all $r$-subsets of $[n]$ containing the vertex $n$. We denote this graph by $H_{n, r}$.

Proposition 13. Let $H$ be the complete $r$-uniform on $[n]$ centered at vertex $n$. Then we have:

$$
P\left(H_{n, r} ; p, q, \boldsymbol{t}\right)=q^{n}+\sum_{S: n \in S \subseteq[n],|S| \geq r} p\left(t_{S}-1\right)(q-1)^{n-|S|}
$$

where $t_{S}=\prod_{T: n \in T \subseteq S,|T|=r} t_{T}$.

Proof. The term $q^{n}$ enumerates all colorings. The summation enumerates colorings which have some primary edges. Hence such colorings are enumerated twice, one with weight one, and once with weight $t_{S}-1$, so that, after cancellation, we actually enumerate all colorings once with the appropriate weight. If a coloring $f$ has any primary edges, then $f(n) \in[p]$. Let $S$ be the color class of the vertex $n$. Note that there are $p$ choices for this color. Also, all the remaining vertices of $H_{n, r}$ must be colored with a color that is not $p$. Otherwise, there is some $r$-edge containing $n$, this vertex, and $r-2$ other vertices of $S$, hence forming a primary edge we failed to count. So there are $(q-1)^{n-|S|-1}$ ways to choose to color the vertices of $V \backslash S$. After summing over all choices of $S$, we achieve our result. 
Setting $t_{e}=t$ for all hyperedges $e$, we obtain:

\section{Corollary 14.}

$$
P\left(H_{n, r} ; p, q, t\right)=q^{n}+\sum_{k=r-1}^{n-1}\left(\begin{array}{c}
n-1 \\
k
\end{array}\right) p\left(t^{\left(\begin{array}{c}
n-1 \\
k
\end{array}\right)}-1\right)(q-1)^{n-k-1} .
$$

Another interesting class of hypergraphs is the class of sunflowers. A sunflower hypergraph $H$ is a hypergraph with a set $S \subseteq V$ such that $S \subseteq e$ for every hyperedge $e$, and $\{e \backslash S: e \in E(H)\}$ is a collection of pairwise disjoint sets. Note that we require $H$ to have no parallel hyperedges. We refer to the vertices of $S$ as seeds.

Proposition 15. Let $H$ be a sunflower hypergraph with hyperedge set $\left\{e_{1}, \ldots, e_{\ell}\right\}$, and no isolated vertices. Then we have We have

$$
P(H ; p, q, \boldsymbol{t})=q^{n}+\sum_{\emptyset \neq J \subseteq[\ell]} p\left(t_{J}-1\right) \prod_{e \notin E_{J}}\left(q^{|e|-s}-1\right)
$$

where $s$ is the number of seeds of $H$, and $t_{J}=\prod_{i \in J} t_{e_{i}}$.

Proof. Fix some non-empty set $J \subseteq[\ell]$, and consider all colorings $f$ for which $P(f)=E_{J}$. Since the hypergrph is a sunflower, all primary hyperedges have the same primary color. There are $p$ ways to choose this color. For a non-primary hyperedge $e$, we have already fixed a coloring of the seeds $S \subset e$, and we just need to color $e \backslash S$. There are $q^{|e|-s}-1$ ways to color the remaining vertices of $e$ such that $e$ is not primary. Since $H$ is a sunflower, the vertices of $e \backslash S$ are not on any other hyperedge, we end up counting all colorings by taking the the product, over all $e \notin E_{J}$ of the ways to color the non-primary edges. The term $t_{J}$ comes from the fact that $E_{J}$ is the set of primary edges. Note that $q^{n}$ counts all colorings, so we are counting the colorings where $E_{J}$ is primary twice, once with weight 1 , and once with weight $t_{S}-1$. After cancellation, we have succeeded in counting all colorings of $H$ by the a ppropriate weights.

Corollary 16. Suppose $S_{r, \ell, s}$ is the $r$-uniform sunflower hypergraph with $\ell$ hyperedges, $s$ seeds, and no isolated vertices. Then we have

$$
P\left(S_{r, \ell, s} ; p, q, t\right)=q^{n}+\sum_{k=1}^{\ell} p\left(\begin{array}{l}
\ell \\
k
\end{array}\right)\left(t^{k}-1\right)\left(q^{r-s}-1\right)^{\ell-k}
$$

\section{Future Work}

We have chosen not to investigate computation complexity questions in this paper. One question is to write an algorithm to determine the coefficients of $\xi(H ; x, y, z)$ or $P(H ; p, q)$. For graphs with bounded tree-width, polynomial time algorithms exist [AGM10]. It seems techniques in this area should work for hypergraphs. We ask the following question: given integers $m, p$ and $k$, is there a polynomial time algorithm for computing the multivariate 
chromatic polynomial of a hypergraph $H$, provided the maximum size of an hyperedge of $H$ is at most $m$, there are at most $p$ pairwise parallel hyperedges, and $H$ has hypertreewidth at most $k$ ? Is the runtime of such an algorithm a polynomial in $n, m, p$ and $k$ ? The notion of hypertree-width was discovered by Gottlob et al [GLS02]. Note that the purpose behind including parameters $p$ and $d$ is to ensure that there is a polynomial bound on the number of hyperedges of $H$, and hence the number of variables $t_{e}$.

Another common question is to determine the computational complexity of evaluating the polynomials in general at a given point. However, this question does not make sense for multivariate polynomials, so we have to consider 'labeled' versions of our polynomials, such as what is studied by Averbouch, Godlin and Makowsky [AGM10]. We note that one can take such an approach. Rather than define labeled variants of the hyperedge elimination polynomial, we state the question only for the hyperedge elimination polynomial. Given a point $\left(x_{0}, y_{0}, z_{0}\right)$, and an integer $d$, what is the complexity of determining $\xi\left(H ; x_{0}, y_{0}, z_{0}\right)$ for all $d$-uniform hypergraphs $H$ ? We restrict the question to $d$-uniform hypergraphs, because otherwise we already know that $\xi\left(H ; x_{0}, y_{0}, z_{0}\right)$ is at least $\# P$-hard to evaluate for all but a finite choice of $\left(x_{0}, y_{0}, z_{0}\right)$, because this is already true for graphs. However, for $d>2$, this does not immediately follow from the case of graphs. The proof techniques from graph polynomials seem like they are still applicable.

Finally, there is a notion of mixed-hypergraph coloring, which has been studied extensively by Voloshin [Vol03]. A mixed hypergraph has two types of hyperedges, called type $C$ and type $D$. For a mixed hypergraph, a coloring is proper if no hyperedge of type $C$ is monochromatic, and no hyperedge of type $D$ is rainbow. Recall that an hyperedge is rainbow if each of its vertices get distinct colors. There is a chromatic polynomial for mixed hypergraphs, and it can be computed usings a recursive algorithm, known as splitting-contraction. One could naturally consider a mixed hypergraph analogue of the multivariate chromatic polynomial. It would be interesting to see if there is any deletion-contraction-extraction analogue of splitting-contraction in this case, and if there are interesting evaluations for such polynomials.

\section{References}

[AGM10] Ilia Averbouch, Benny Godlin, and J. A. Makowsky, An extension of the bivariate polynomial, European J. Combin. 31 (2010), no. 1, 1-17. MR 2552585

[Ber73] Claude Berge, Graphs and hypergraphs, North-Holland Publishing Co., Amsterdam, 1973, Translated from the French by Edward Minieka, North-Holland Mathematical Library, Vol. 6. MR 0357172 (50 \#9640)

[BL46] G. D. Birkhoff and D. C. Lewis, Chromatic polynomials, Trans. Amer. Math. Soc. 60 (1946), 355-451. MR 0018401 (8,284f)

[BO92] Thomas Brylawski and James Oxley, The Tutte polynomial and its applications, Matroid applications, Encyclopedia Math. Appl., vol. 40, Cambridge Univ. Press, Cambridge, 1992, pp. 123-225. MR 1165543 (93k:05060) 
[BR99] Béla Bollobás and Oliver Riordan, A Tutte polynomial for coloured graphs, Combinatorics, Probability, and Computing (1999), no. 8, 45-93.

[Cra69] Henry H. Crapo, The Tutte polynomial, Aequationes Math. 3 (1969), 211-229. MR 0262095 (41 \#6705)

[DPT03] Klaus Dohmen, André Pönitz, and Peter Tittmann, A new two-variable generalization of the chromatic polynomial, Discrete Math. Theor. Comput. Sci. 6 (2003), no. 1, 69-89 (electronic). MR 1996108 (2004j:05053)

[EMM11a] Joanna A. Ellis-Monaghan and Criel Merino, Graph polynomials and their applications I: The Tutte polynomial, Structural Analysis of Complex Networks (Matthias Dehmer, ed.), Birkhäuser Boston, 2011, 10.1007/978-0-8176-47896_9, pp. 219-255.

[EMM11b] _ Graph polynomials and their applications II: Interrelations and interpretations, Structural Analysis of Complex Networks (Matthias Dehmer, ed.), Birkhäuser Boston, 2011, 10.1007/978-0-8176-4789-6_10, pp. 257-292.

[GJ10] Leslie Goldberg and Mark Jerrum, Approximating the partition function of the ferromagnetic potts model, Automata, Languages and Programming (Samson Abramsky, Cyril Gavoille, Claude Kirchner, Friedhelm Meyer auf der Heide, and Paul Spirakis, eds.), Lecture Notes in Computer Science, vol. 6198, Springer Berlin / Heidelberg, 2010, pp. 396-407.

[GLS02] Georg Gottlob, Nicola Leone, and Francesco Scarcello, Hypertree decompositions and tractable queries, J. Comput. System Sci. 64 (2002), no. 3, 579-627, Special issue on PODS 1999 (Philadelphia, PA). MR 1915026 (2003g:68052)

[HL72] C. J. Heilman and E. H. Lieb, Theory of monomer-dymer systems, Comm. Math. Phys (1972), no. 28, 190-232.

[Sok05] Alan D. Sokal, The multivariate Tutte polynomial (alias Potts model) for graphs and matroids, Surveys in combinatorics 2005, London Math. Soc. Lecture Note Ser., vol. 327, Cambridge Univ. Press, Cambridge, 2005, pp. 173 226. MR 2187739 (2006k:05052)

[Tut47] W. T. Tutte, A ring in graph theory, Proc. Cambridge Philos. Soc. 43 (1947), 26-40. MR 0018406 (8,284k)

[Tut84] _ Graph theory, Encyclopedia of Mathematics and its Applications, vol. 21, Addison-Wesley Publishing Company Advanced Book Program, Reading, MA, 1984, With a foreword by C. St. J. A. Nash-Williams. MR 746795 (87c:05001)

[Vol03] Vitaly Voloshin, Coloring mixed hypergraphs: some results and open problems, Rend. Sem. Mat. Messina Ser. II 9(25) (2003), 237-244 (2004). MR 2121479

[Zas92] Thomas Zaslavsky, Strong Tutte functions of matroids and graphs, Trans. Amer. Math. Soc. 334 (1992), no. 1, 317-347. MR 1080738 (93a:05047) 